\title{
Link-to-System Level Interface Solutions in Multistate Channels for 3GPP LTE Wireless System
}

\author{
Ricardo B. Santos ${ }^{\dagger}$, Walter C. Freitas Jr., Elvis M. G. Stancanelli and Francisco Rodrigo P. Cavalcanti
}

\begin{abstract}
In this paper we present a Link-to-System (L2S) level interface solution able to deal with multistate wireless channels, like when techniques such Multiple Input Multiple Output (MIMO) antennas and Orthogonal Frequency Division Multiplexing (OFDM) are considered. A physical layer model towards Long Term Evolution (LTE) of 3rd. Generation Partnership Project (3GPP) was used for evaluation of Effective SNR Mapping (ESM)-based solutions, when we have been assuming several Modulation and Coding Schemes (MCSs) and wireless channel models. The numerical results showed that the use of Exponential ESM (EESM) is suitable to the next generation of Orthogonal Frequency Division Multiple Access (OFDMA) wireless systems.
\end{abstract}

Keywords-L2S interface, multistate channels, OFDMA, MIMO

\section{INTRODUCTION}

The Fourth Generation (4G) of the wireless communication system aims to provide a wide variety of services, each one with different Quality of Service (QoS) requirements, e.g., from high quality voice communication up to wireless multimedia services. These services should be provided to anyone, in anyplace and at anytime, following the Always Best Connected (ABC) concept in a multiple Radio Access Network (RAN), what motivate to pursue high data rates in the air interface with high spectral efficiency.

In order to achieve high data rates in the air interfaces the use of Multiple Input Multiple Output (MIMO) antennas in both transmitter and receiver is being considered for the $4 \mathrm{G}$ systems. Regarding the spectral efficiency issue, the access through Orthogonal Frequency Division Multiple Access (OFDMA) is one of the main strategies proposed. Due to its intrinsic property of converting a broadband frequency selective radio channel into a set of orthogonal narrowband frequency-flat radio channels, and its computationally efficient implementation based on the Fast Fourier Transform (FFT), Orthogonal Frequency Division Multiplexing (OFDM)-based transmission has been showing to be a suitable air interface candidate for $4 \mathrm{G}$ systems. This scheme fits well to the challenging requirements imposed by $4 \mathrm{G}$ systems: on the one hand, high granularity in data rates and high spectral efficiency in the downlink are required while, on the other hand, simple but effective transmission techniques are desired for the uplink to enable for low-cost User Equipments (UEs).

$\dagger$ Student is supported by a scholarship granted by FUNCAP. This work was supported by a grant from Ericsson of Brazil - research branch under ERBB/UFC.18 technical cooperation contract. The authors are in Wireless Telecommunication Research Group - GTEL/Federal University of Ceará UFC. E-mails:\{brauner,walter,emiguel,rodrigo\}@gtel.ufc.br.
The research on $4 \mathrm{G}$ has been taking place in the academia and industry. In the current phase of the forecast performance results, investigations are performed using computer simulation, which is a controlled execution of models of real wireless networks. A suitable approach to implement such simulations consists on the separation of its functionalities into two levels handled by distinct simulators: link- and system-level.

Link-level simulators consider just one pair transmitter-receiver, and here we are interested in user-scale metrics such as Bit Error Rate (BER) and Block Error Rate (BLER). Instead, the interest of system-level simulators resides in the evaluation of the multiple users sharing the same radio resources and the impact on the network performance as well. To match these two approaches, an interface solution is necessary. Well-known solutions, such as the Actual Value Interface (AcVI) [2],[3], are suitable for one-state wireless channels, e.g. Additive White Gaussian Noise (AWGN) and flat fading channels. Because $4 \mathrm{G}$ systems are expected to consider MIMO and/or OFDMA techniques, a multistate wireless channel takes place and the early solutions are no more suitable.

In this paper, we evaluated some possible Link-to-System (L2S) level interface solutions when considering multistate wireless channels. This evaluation was done assuming the 3rd. Generation Partnership Project (3GPP) Long Term Evolution (LTE), which is OFDMA-based, and realistic radio channels as well. Multiple Modulation and Coding Scheme (MCS) allow performing link adaptation.

The paper is organized as follows. In Section II, we describe 3GPP LTE wireless system. Section III describes L2S interface solutions in multistate channels when considering OFDM techniques. Section IV presents our simulation results. Finally, in Section V, some conclusions are drawn.

\section{3GPP LTE SYSTEM}

3GPP is now in process of standardization of the so-called LTE for Third Generation (3G) radio access systems. The main goals are to increase data rates, spectral efficiency, and coverage, and to reduce latency, besides achieving the following targets and requirements [1]:

- Peak data rates exceeding $100 \mathrm{Mbps}$ for the downlink direction and 50 Mbps for the uplink direction;

- Users' mean throughput improved by factors 2 and 3 for uplink and downlink respectively;

- User's cell-edge throughput improved by a factor 2 for uplink and downlink; 
- Uplink and downlink spectrum efficiency improved by factors 2 and 3 respectively;

- Significantly reduced control-plane latency;

- Reduced cost for operator and end user;

- Spectrum flexibility, enabling deployment in many different spectrum allocations.

In the radio interface of LTE, OFDM is being considered as well as MIMO antennas. In the downlink direction, LTE considers a frequency structure based on a large number of individual sub-carriers distanced of $15 \mathrm{kHz}$. Sub-frame duration is set as short as $0.5 \mathrm{~ms}$ in order to minimize the radio-interface latency. In order to handle different delay spreads and corresponding cell sizes with a modest overhead, the OFDM cyclic prefix length can assume two different values. The shorter cyclic prefix of $4.7 \mu \mathrm{s}$ is enough to handle the delay spread for most unicast scenarios. With the longer cyclic prefix of $16.7 \mu$ s, very large cells, up to and exceeding $120 \mathrm{~km}$ cell radius, with large amounts of time dispersion, can be handled. In this case, the length is extended by reducing the number of OFDM symbols in a sub-frame.

Link adaptation, i.e. Adaptive Modulation and Coding (AMC), is also employed in LTE. AMC with three modulation schemes - Quadri-Phase Shift Keying (QPSK), 16 Quadrature Amplitude Modulation (16QAM) and 64 Quadrature Amplitude Modulation (64QAM) - and channel coding rates based on a rate $1 / 3$ turbo coder are applied to the shared data channel. By default, the same MCS will be considered for all the resource blocks of the same Medium Access Control (MAC) Protocol Data Unit (PDU) allocated on a Physical Resource Block (PRB) to a UE during one Transmission Time Interval (TTI). The use of power and modulation adaptation per resource block is left for further specification in LTE.

Since the 3GPP LTE considers turbo coder for channel coding, it is necessary a soft-output demapper to all modulation schemes. For QPSK the soft information associated from each bit can be calculated by the in-phase and in-quadrature components. However, in the case of 16QAM and 64QAM constellations, each axis carries more than one bit and the metric functions to determine the soft information for each bit in the soft output demapper is quite complicated. Here we will consider the simplified soft-output demapper from [8], since the complexity of the demapper becomes at almost the same for all possible constellations. For the in-phase bits of a 16QAM constellation (the same applies to the in-quadrature analysis), the soft information can be simplfied by:

$$
\begin{aligned}
& D_{I, 1} \cong-y_{I}[i] \\
& D_{I, 2} \cong 2-\left|y_{I}[i]\right|,
\end{aligned}
$$

where $y_{I}[i]$ is the $i^{t h}$ in-phase component of the received vector. Similar expressions can be obtained from a 64QAM constellation.

$$
\begin{aligned}
& D_{I, 1} \cong-y_{I}[i] \\
& D_{I, 2} \cong\left|y_{I}[i]\right|-4 \\
& D_{I, 3} \cong|| y_{I}[i]|-4|-2 .
\end{aligned}
$$

The $D_{Q, k}$ for the in-quadrature bits are the same of the in-phase with $y_{I}[i]$ replaced by $y_{Q}[i]$.

\section{LINK-TO-SySTEM InTERFACE SOLUTIONS}

To achieve the promised high data rates with high spectral efficiency for the next $4 \mathrm{G}$ wireless systems in MAC and Physical (PHY) layers, OFDM and/or MIMO techniques have been being considered. On the one hand, the MIMO antennas strategy can increase the system capacity almost linearly with the lower number of antennas; on the other hand OFDM turns a broadband wireless channel in multiple narrowband channels making the equalization easier. Additionally, with OFDMA the system can capture user diversity, since a weak subcarrier to a given user could be in a good situation to other user. However, with such advanced techniques the analysis of the channel becomes hard, since we must take into account the state of each sub-channel or subcarrier. The multistate approaches must take place.

In general, L2S interfaces must be designed to allow a high accuracy on the exchange of metrics between link- and system-level simulators, so that the link-level behavior is reflected on the system-level part, avoiding their simultaneous operation and thus allowing a complete simulation in a feasible way. Well-known solutions can provide L2S interface for several network architectures with distinct trade-offs between accuracy and complexity. Solutions like Average Value Interface (AVI) and AcVI are specifically conceived for one-state channels. For OFDMA-based systems, the challenge in L2S interfaces is to make the link performance assessment at the system-level ready, regarding the channel conditions on every subcarrier.

\section{A. Effective SNR Mapping (ESM)}

An attractive way for L2S investigations in multistate channels consists on the Effective SNR Mapping (ESM) approach [7]. The principle of ESM can be separated in two stages (Fig. 1): compression and quality mapping. In the first stage, an effective Signal-to-Noise Ratio (SNR) is found comprising all multiple states of the channel. In the latter, the effective SNR value is mapped to a performance metric such as BLER or Frame Erasure Rate (FER) in a one-state channel, e.g. AWGN channel. This result is proved in [7], as shown in Appendix A.

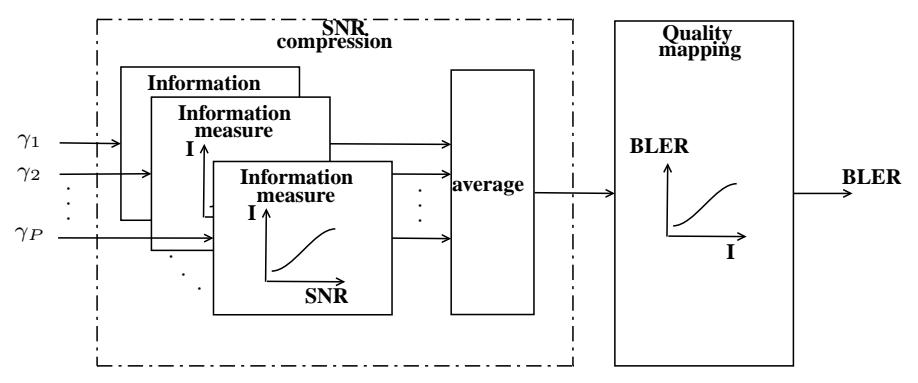

Fig. 1. Principle of Effective SNR Mapping.

The main goal of the SNR compression stage is to compact information about the multiple states of the channel into a 
single metric - the effective SNR, $\gamma_{\text {eff }}$ :

$$
\gamma_{e f f}=\alpha_{1} \cdot I^{-1}\left[\sum_{n=1}^{N} I\left(\frac{\gamma_{n}}{\alpha_{2}}\right) p_{n}\right]
$$

where $I(\cdot)$ is the information measure [5], $(\cdot)^{-1}$ is the inverse function, $N$ is the number of states of the channel (number of subcarries in the OFDMA case) each one with probability $p_{n}, \gamma_{n}$ is the SNR of the $n^{t h}$ subcarrier, $\alpha_{1}$ and $\alpha_{2}$ are parameters that allow to adapt the model to the intended MCS curve. Information measures describing a sigmoidal curve are suitable for modeling practical channel codes since the amount of information that a channel can pass should be bounded [5].

After the compression stage, either an AWGN curve [6], [4] or a curve derived from data samples can supply the quality mapping stage. Few versions of ESM were published [7], [5], [6], such as Capacity ESM (CESM), Cutoff Rate ESM (CRESM), Linear ESM (LiESM), Exponential ESM (EESM), Logarithmic ESM (LESM) and Mutual-Information ESM (MIESM). Some of them are described in the following, in which the quality mapping functions distinguish from each other about the information measure.

The LiESM is the simplest information measure. The mapping just considers the SNR of the $n^{\text {th }}$ subcarrier as the information measure

$$
I_{\operatorname{LiESM}}\left(\gamma_{n}\right)=\gamma_{n}
$$

Other information measures consider the logarithm and exponential functions like

$$
\begin{aligned}
& I_{L E S M}\left(\gamma_{n}\right)=\log \left(\gamma_{n}\right) \\
& I_{E \operatorname{ESM}}\left(\gamma_{n}\right)=1-\exp \left(-\gamma_{n}\right) .
\end{aligned}
$$

For EESM, replacing the information measure (6) in (3) we have

$$
\begin{aligned}
\gamma_{e f f} & =\alpha_{1} \cdot I^{-1}\left[\sum_{n=1}^{N}\left\{1-\exp \left(\frac{-\gamma_{n}}{\alpha_{2}}\right)\right\} p_{n}\right] \\
& =\alpha_{1} \cdot I^{-1}\left[\sum_{n=1}^{N} p_{n}-\sum_{n=1}^{N} p_{n}\left\{\exp \left(\frac{-\gamma_{n}}{\alpha_{2}}\right)\right\}\right] \\
& =\alpha_{1} \cdot I^{-1}\left[1-\sum_{n=1}^{N} p_{n}\left\{\exp \left(\frac{-\gamma_{n}}{\alpha_{2}}\right)\right\}\right] .
\end{aligned}
$$

Assuming that the multiple states are equiprobable, the effective SNR can be given simply by:

$$
\gamma_{e f f}=-\alpha_{1} \ln \left[\frac{1}{N} \sum_{n=1}^{N} \exp \left(-\frac{\gamma_{n}}{\alpha_{2}}\right)\right] .
$$

In the process of the L2S interface specification, the scaling factor parameters $\alpha_{1}$ and $\alpha_{2}$ should be adjusted to increase the accuracy of the composition of information measure and quality mapping. These parameters should be related with the modulation cardinality and the channel coding scheme. Thus, each pair of parameters is stipulated to just one MCS.

\section{RESUlts}

In this section, simulation results related to the previously exposed L2S interface solutions are presented. The channel models simulated were the 3GPP Typical Urban (TU) and Hilly Terrain (HT) models. To generate the fading effect for each channel model, we perform 40 independent realizations each with 10 TTIs of $0.5 \mathrm{~ms}$ preserving a same average of sub-carrier SNRs. Afterwards, this average SNR value was variated according to a range from -5 to $20 \mathrm{~dB}$, stepping by $1 \mathrm{~dB}$. In each TTI, 7 OFDM symbols are transmitted. The LTE sub-frame is defined as 7 OFDM symbols in sequence in one subcarrier. In this work, the BLER is calculated as the probability of sub-frame error on all subcarriers for all realizations of this channel.

An AWGN simulation for each MCS is performed in the sense to give the target of the mapping. The MCSs are QPSK, 16QAM and 64QAM. Turbo coding is composed of two parallel 8 -state constituent encoders, coding rate of $1 / 3$, and the decoder performs a SOVA-based algorithm [9] with 8 iterations.

In Fig. 2, results for the LiESM mapping are presented besides the AWGN curve. Note that in this mapping we have a direct correspondence between the average SNR value and $\gamma_{e f f}$. We can observe that different BLERs were obtained for the same effective $\gamma_{e f f}$. Thus, the LiESM is not a suitable L2S interface for the simulated channel.

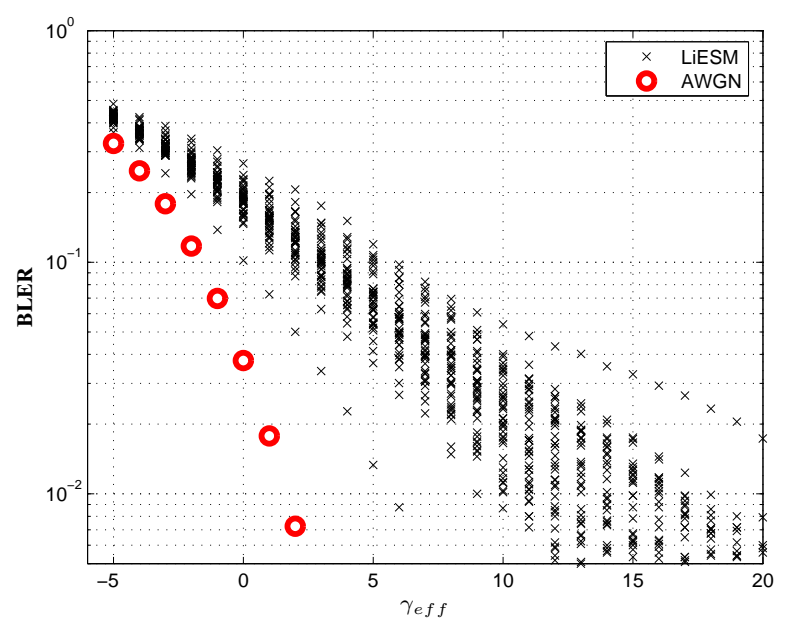

Fig. 2. Linear ESM in QPSK and 3GPP TU channel.

In Fig. 3, results for the LESM mapping are shown. Alike using LiESM, the LESM mapping cannot represent the actual state of the channel and is also not an appropriate L2S interface solution for the simulated channel.

For the EESM mapping, the $\alpha_{1}$ and $\alpha_{2}$ (see equation (8)) are key parameters for a suitable representation of the channel state. Fig. 4 shows the impact of an inappropriate parameter choice for the QPSK modulation. For the subsequent results, an extensive search for the appropriate parameters was made assuming $\alpha_{1}=\alpha_{2}=\alpha$, whose chosen values are presented in Table I. 


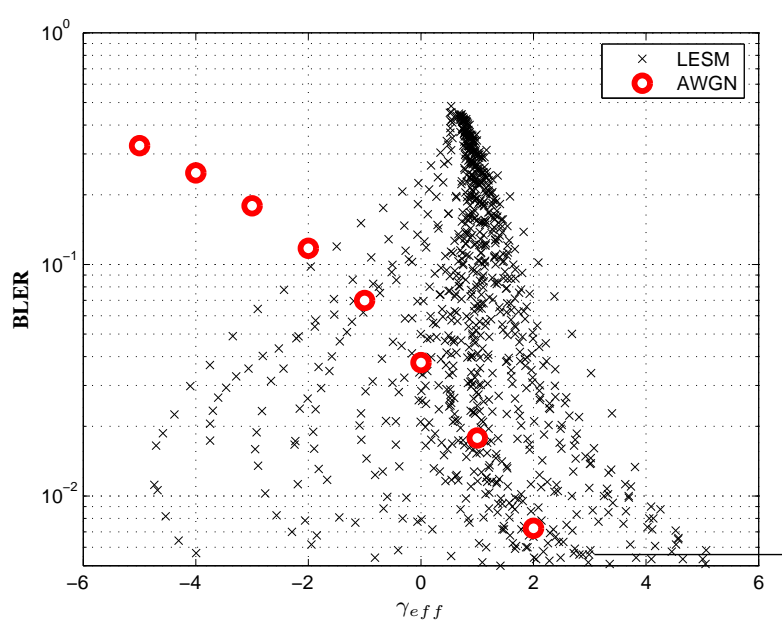

Fig. 3. Logarithmic ESM in QPSK and 3GPP TU channel.

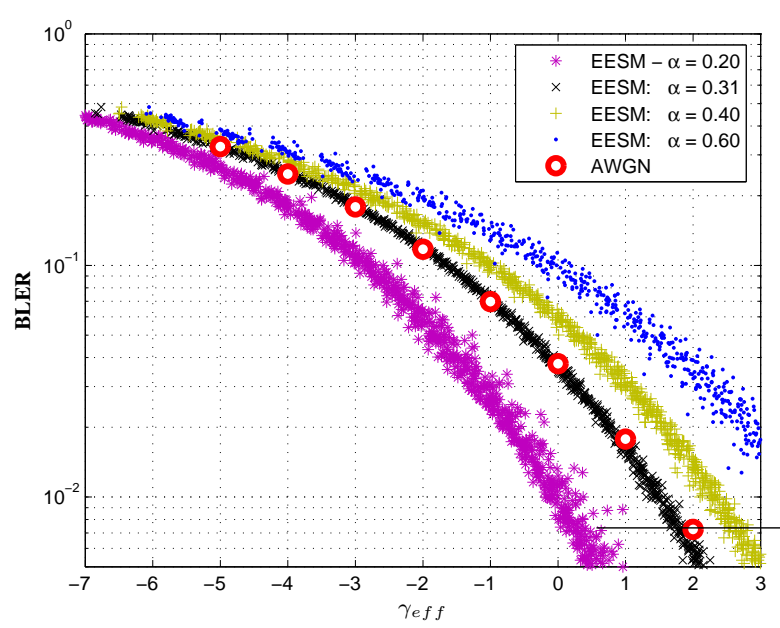

Fig. 4. Impact of the $\alpha$ parameter choice in Exponential ESM for QPSK and 3 GPP TU channel.

TABLE I

EESM MAPPING PARAMETERS.

\begin{tabular}{c|c}
\hline \hline Modulation & $\alpha$ \\
\hline \hline QPSK & 0.311 \\
\hline 16QAM & 1.04 \\
\hline 64QAM & 2.2 \\
\hline \hline
\end{tabular}

In Fig. 5, Fig. 6 and Fig. 7, the results for the EESM mapping are presented for the QPSK, 16QAM and 64QAM, respectively, in the 3GPP TU channel. Therefore we can see that the EESM mapping is able to represent the channel characteristic very well.

In Fig. 8, 9 and 10, the results for the EESM mapping are presented for the QPSK, 16QAM and 64QAM respectively in the 3GPP HT channel. The results are almost identical to the TU channel ones and used the same $\alpha$ values. This enforces the independence between the mapping $\alpha$ parameter and the channel model.

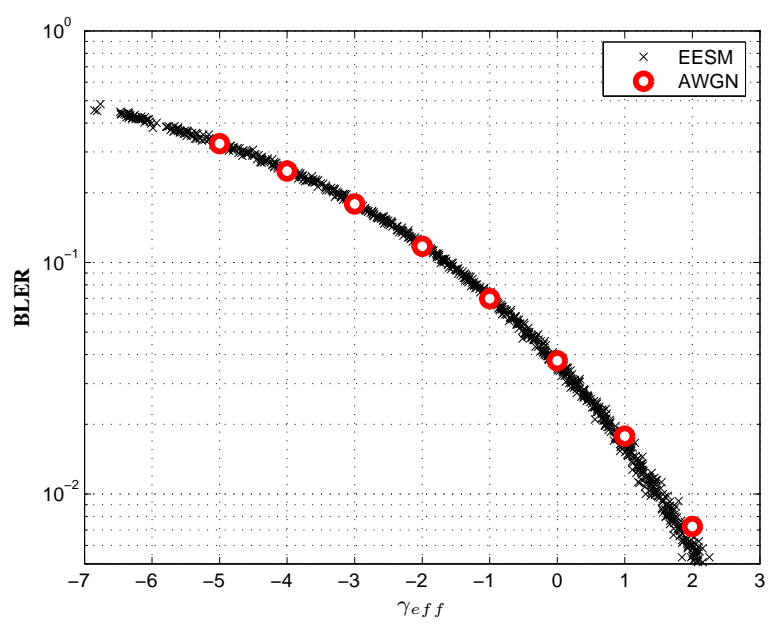

Fig. 5. Exponential ESM in QPSK and 3GPP TU channel.

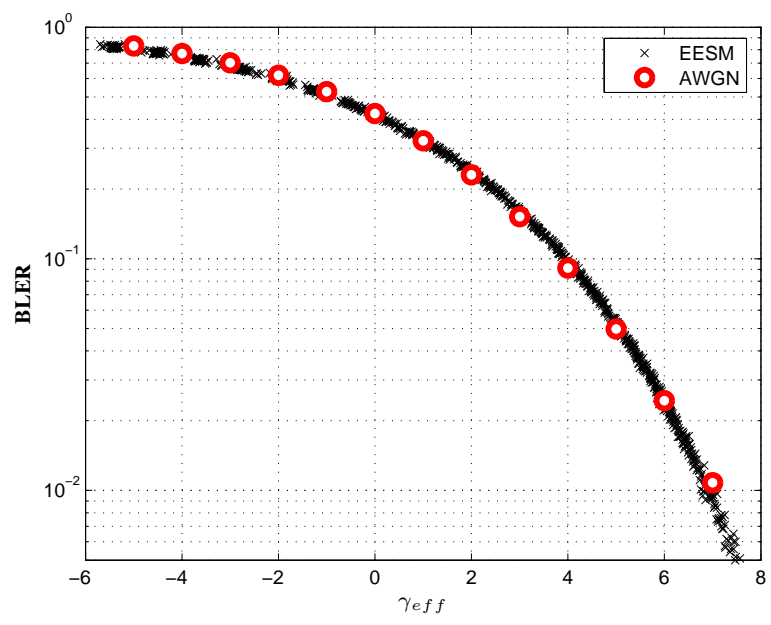

Fig. 6. Exponential ESM in 16QAM and 3GPP TU channel.

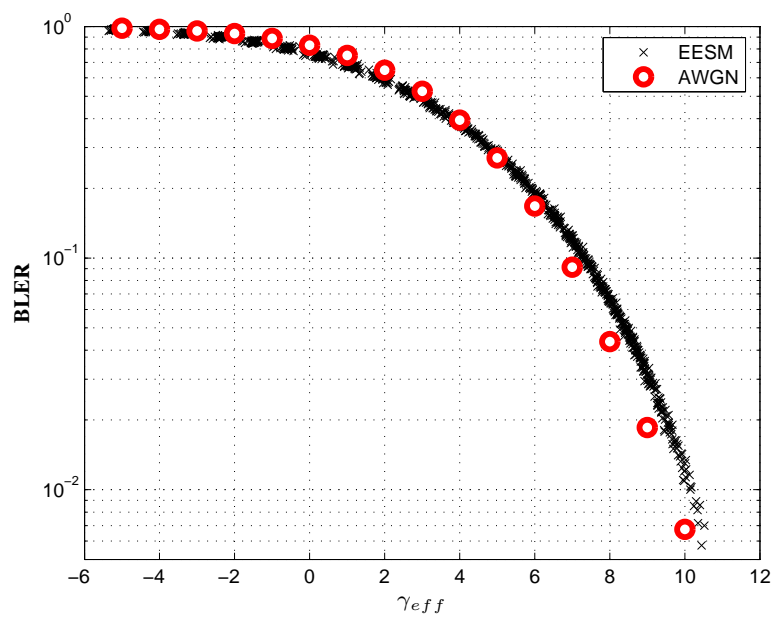

Fig. 7. Exponential ESM in 64QAM and 3GPP TU channel.

\section{Conclusions}

In this paper, we presented some possible solutions considering the L2S level interface in multistate wireless 


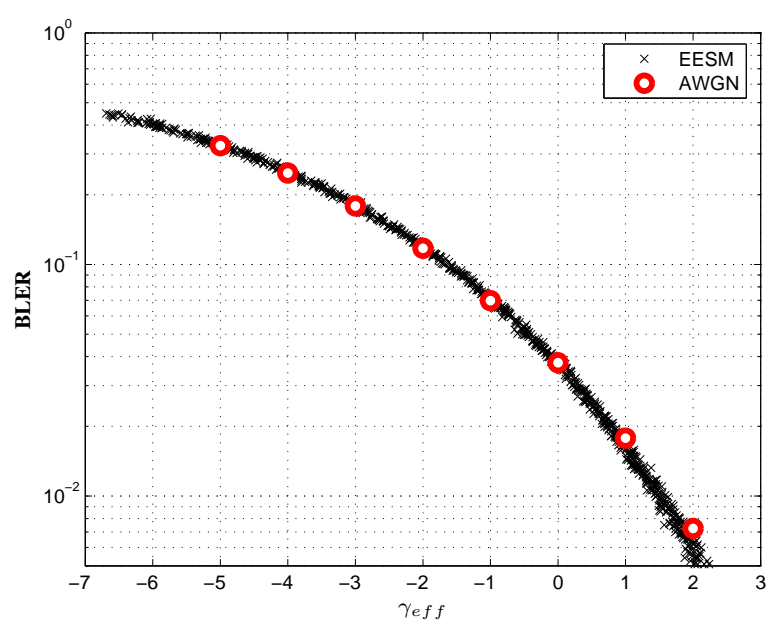

Fig. 8. Exponential ESM in QPSK and 3GPP HT channel.

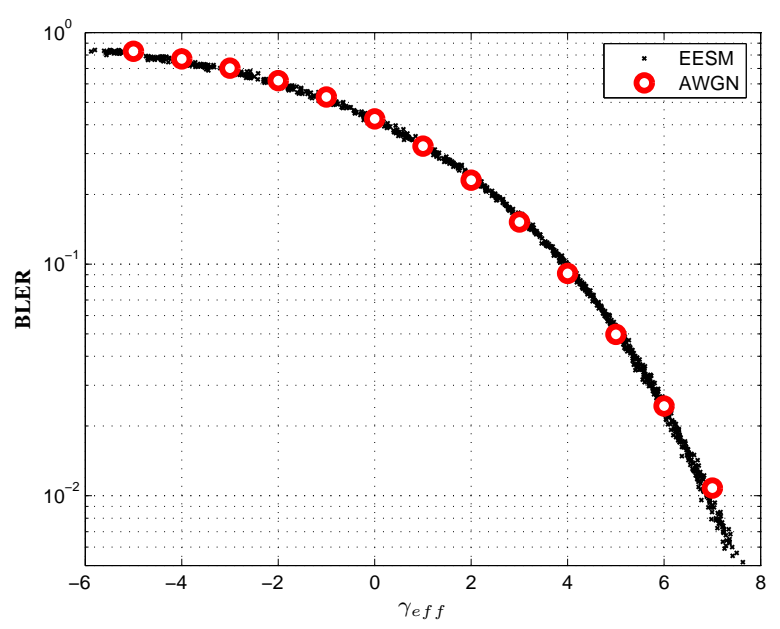

Fig. 9. Exponential ESM in 16QAM and 3GPP HT channel.

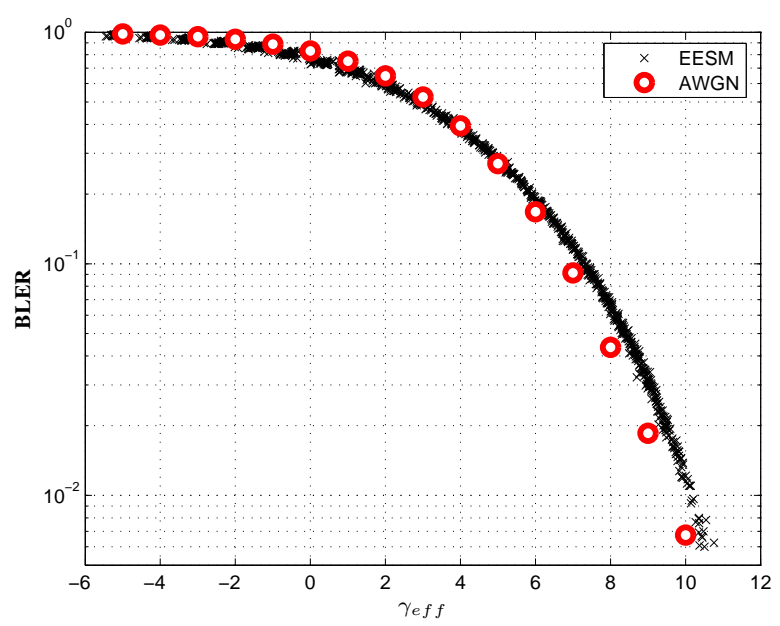

Fig. 10. Exponential ESM in 64QAM and 3GPP HT channel. channels for systems that consider OFDMA. Ours results are based on the 3GPP LTE wireless system that is being considered as a candidate for $4 \mathrm{G}$ wireless systems. It was shown that EESM is a very accurate L2S level interface solution for all MCS and channel models of the 3GPP LTE system. However, the adjust step of the $\alpha$ is crucial to achieve an accurate result.

The use of the mutual-information is a promising improvement in the sense that the MCSs are taken into account in the information measure, differently from the EESM in which the MCS is taken into account in the scaling factor parameters. However, the calculation of the mutual information for high modulation orders and decoding algorithms may be a difficult task itself.

\section{REFERENCES}

[1] 3GPP, TR 25.913, Requirements for Evolved UTRA and Evolved UTRAN, www.3gpp.org.

[2] S. Hamaldinen et.al.- A Novel Interface Between Link and System Level Simultations. Proceedings ACTS Mobile Communications Summit 97, vol 2., October 1997, Aalborg, Denmark. pp 599-604.

[3] Olofsson et.al.. Improved Interface Between Link Level and System Level Simulations Applied to GSM. Record of 6th IEEE International Conference on Universal Personal Communications. vol. 1. October 1997. pp. 79-83.

[4] Esa Tuomaala and Haiming Wang, Effective SINR approach of link to system mapping in OFDM multi-carrier mobile network, 2nd International Conference on Mobile Technology, Applications and Systems, 2005.

[5] S. Shawn Tsai and Anthony C. K. Soon, Effective-SNR mapping for Modelling Frame Error Rates in Multiple-state Channels, Ericsson, 3GPP2-C30-20030429-010.

[6] K. Brueninghaus, D. Astely, T. Salzer, S. Visuri, A. Alexiou, S. Karger and G.-A. Seraji, Link performance models for system level simulations of broadband radio access systems, IEEE 16th International Symposium on Personal, Indoor and Mobile Radio Communications, vol.04, pp. 2306-2311, 2005.

[7] Mathias Pauli, Udo Wachsmann and Shiau-He (Shawn) Tsai, Quality Determination for a Wireless Communications Link, United States Patent Application Publication, 2004, US2004/0219883 A1.

[8] F. Tosato and P. Bisaglia, Simplified Soft-Output Demapper for Binary Interleaved COFDM with Application to HIPERLAN/2, IEEE International Conference on Communications, vol .02, pp. 664-668, 2002

[9] P. Robertson, E. Villebrun and P. Hoeher, A comparison of optimal and sub-optimal MAP decoding algorithms operating in the log domain, IEEE International Conference on Communications, vol. 02, June 1995.

\section{APPENDIX A}

Either OFDM or MIMO techniques considered in MAC and PHY layers result in a multistate communication link, since the transmission of a code word using a specified MCS the channel is varying during a TTI. In this case, for example, the SNR has to be represented as a vector $\vec{\gamma}=\left[\gamma_{1}, \gamma_{2}, \ldots, \gamma_{n}\right]$, in which $n$ is the instance experienced by the multistate channel.

Using the compression stage it is possible to express the performance of a coded transmission over a multistate channel in terms of the AWGN performance. This outstanding result follows of the theorem using the Union Chernoff bound.

Theorem A: The effective SNR value $\gamma_{\text {eff }}$ of an equivalent one-state channel is equal of the one on the multistate channel:

$$
P_{e}\left(\gamma_{e f f}\right)=P_{e}(\vec{\gamma}),
$$

where $P_{e}(\cdot)$ is the error probability for a certain channel. 
Proof [7]: The union bound for a coded transmission using Maximum Likelihood (ML) detection is upper bounded by

$$
P_{e}\left(\frac{E_{s}}{N_{0}}\right) \leq \sum_{d=d_{\min }}^{\infty} \delta_{d} P_{2}\left(d, \frac{E_{s}}{N_{0}}\right)
$$

where $P_{e}\left(\frac{E_{s}}{N_{0}}\right)$ is the error probability of deciding per a wrong code word to a channel state characterized by a SNR equals to $\frac{E_{s}}{N_{0}}$ to a mean energy per symbol $E_{s}$ and noise power density $N_{0}, d_{\text {min }}$ is the minimum Hamming distance, $\delta_{d}$ represents the Hamming distance distribution and $P_{2}\left(d, \frac{E_{s}}{N_{0}}\right)$ is the Pairwise Error Probability (PEP). The PEP to a BPSK transmission over AWGN channel with ML detection is given by

$$
P_{2}\left(d, \frac{E_{s}}{N_{0}}\right)=Q\left(\sqrt{2 \frac{E_{s}}{N_{0}} d}\right)=Q(\sqrt{2 \gamma d})=P_{2}(d, \gamma),
$$

where

$$
Q(x)=\frac{1}{2} \operatorname{erfc}\left(\frac{x}{\sqrt{2}}\right)=\frac{1}{\sqrt{2 \pi}} \int_{x}^{\infty} e^{-t^{2} / 2} d t, x \geq 0,
$$

where $Q$-function is a derivative from the complementary gaussian error function $\operatorname{erfc}(x)$. Using the upper bound to the $Q$-function

$$
Q(x) \leq e^{-x^{2} / 2}
$$

Using this upper bound in the PEP definition

$$
P_{2}(d, \gamma) \leq e^{-\gamma^{d}}
$$

this upper bound on the PEP is defined as $P_{2, \text { Chernoff }}$. It follows to one state-channel

$$
P_{2, \text { Chernoff }}(d, \gamma)=e^{-\gamma^{d}}=\left(e^{-\gamma}\right)^{d}=\left[P_{2, \text { Chernoff }}(1, \gamma)\right]^{d} \text {. }
$$

This result relates the Chernoff-bound PEP with the symbol error probability (Chernoff-bound (uncoded) PEP). Therefore, the union bound could be upper bounded using the Chernoff-bound PEP

$$
\begin{aligned}
P_{e}(\gamma) \leq \sum_{d=d_{\min }}^{\infty} \delta_{d} P_{2}(d, \gamma) \leq \sum_{d=d_{\min }}^{\infty} \delta_{d}\left[P_{2, \text { Chernoff }}(1, \gamma)\right]^{d} \\
=P_{e, \text { Chernoff }}(\gamma) .
\end{aligned}
$$

The principle of this L2S interface regarding the union Chernoff bound will be explained considering a two-state channel, but the extension to a higher order multistate channel if straightforward. In such two-state channel the SNR is characterized as $\vec{\gamma}=\left[\gamma_{1}, \gamma_{2}\right]$. Consider here that both states are independent each other, and the probability of such states occur be $p_{1}$ and $p_{2}$, respectively and $p_{1}+p_{2}=1$. The PEP averaged over all the possible positions of the symbols of the $d$ difference symbols using the Chernoff-bound is given by

$$
P_{2, \text { Chernoff }}\left(d,\left[\gamma_{1}, \gamma_{2}\right]\right)=\sum_{i=0}^{d}\left(\begin{array}{l}
d \\
i
\end{array}\right) p_{1}^{i} p_{2}^{d-i} e^{-\left(i \gamma_{1}+(d-i) \gamma_{2}\right)},
$$

where $p_{1}^{i} p_{2}^{d-i}$ is the probability that $i$ of the $d$ difference symbols are associated with $\gamma_{1}$ and the other ones with $\gamma_{2}$, this event occurs $i^{d}$ times and $e^{-\left(i \gamma_{1}+(d-i) \gamma_{1}\right)}$ is the Chernoff-bounded PEP of such event.

Using the binomial theorem we can represent the last result as

$$
\begin{aligned}
P_{2, \text { Chernoff }}\left(d,\left[\gamma_{1}, \gamma_{2}\right]\right) & =\sum_{i=0}^{d}\left(\begin{array}{l}
d \\
i
\end{array}\right)\left(p_{1} e^{-\gamma_{1}}\right)^{i}\left(p_{2} e^{-\gamma_{2}}\right)^{d-i} \\
& =\left(p_{1} e^{-\gamma_{1}}+p_{2} e^{-\gamma_{2}}\right)^{d} . \quad(\mathrm{A}-10)
\end{aligned}
$$

This last expression represents the averaged Chernoff-bounded symbol error probability on this two-state channel. Therefore the expression found to one-state channel has to be also valid to this two-state channel, thus,

$$
P_{2, \text { Chernoff }}\left(d,\left[\gamma_{1}, \gamma_{2}\right]\right)=\left[P_{2, \text { Chernoff }}\left(1,\left[\gamma_{1}, \gamma_{2}\right]\right)\right]^{d} \text {. }
$$

To higher order state channels this same result can be achieved using the polynomial theorem, that generalizes the previous result as

$$
P_{2, \text { Chernoff }}(d, \vec{\gamma})=\left[P_{2, \text { Chernoff }}(1, \vec{\gamma})\right]^{d}
$$

In summary, using this approach, the goal is to find an effective SNR value $\gamma_{e f f}$ of an equivalent one-state channel such that the Chernoff-bounded error probability equals the one on a multistate channel, or,

$$
P_{e, \text { Chernoff }}\left(\gamma_{\text {eff }}\right)=P_{e, \text { Chernoff }}(\vec{\gamma}) \text {. }
$$

